\title{
On the Form of the Optimal Measurement for the Probability of Detection
}

\author{
Rafał Wieczorek ${ }^{1}$
}

Received: 4 November 2014 / Accepted: 8 April 2015 / Published online: 28 April 2015

(C) The Author(s) 2015. This article is published with open access at Springerlink.com

\begin{abstract}
We consider the problem of maximizing the probability of detection for an infinite number of mixed states. We show that for linearly independent states there exists a unique simple optimal measurement, generalizing thus a result obtained in finite dimension by Y. Eldar (Phys. Rev. A, 68, 052303:1-052303:4 2003).
\end{abstract}

Keywords Probability of detection - Linearly independent states $\cdot$ Mixed states $\cdot$ Simple measurement

\section{Introduction}

Let $\rho_{1}, \rho_{2}, \ldots$ be (finite or infinite number) quantum states (density matrices) on $\mathbb{B}(\mathcal{H})$ the bounded linear operators on Hilbert space $\mathcal{H}$, of arbitrary dimension, which can occur with some a priori probabilities $\pi=\left(\pi_{1}, \pi_{2}, \ldots\right)$. We want to find, in an optimal way, the state in which the system really is. To this end we perform a measurement (called also strategy) $\mathbb{M}$, by which is meant a sequence $\left(M_{1}, M_{2}, \ldots\right)$ of positive operators from $\mathbb{B}(\mathcal{H})$, such that

$$
\sum_{i=1}^{\infty} M_{i}=\mathbb{1}
$$

where the series is convergent in the weak operator topology on $\mathbb{B}(\mathcal{H})$. A measurement $\mathbb{M}=\left(M_{1}, M_{2}, \ldots\right)$ for which all $M_{i}$ 's are pairwise orthogonal projections is called simple or sharp (see [1]).

Rafał Wieczorek

wieczorek@math.uni.lodz.pl

1 Faculty of Mathematics and Computer Science, Łódź University, ul. S. Banacha 22, 90-238 Łódź, Poland 
If we receive outcome $M_{i}$, we choose state $\rho_{i}$. The probability that the true state is $\rho_{i}$ when measurement will give result $M_{j}$ is given by $\operatorname{tr}\left(\rho_{i} M_{j}\right)$. Thus $\operatorname{tr}\left(\rho_{i} M_{i}\right)$ is the probabilty of guessing correctly state $\rho_{i}$. If our guess is $\rho_{j}$ while the true one is $\rho_{i}$, then we pay penalty $L(i, j)$. Function $L$ is called a loss function. The risk function is defined by the formula

$$
R_{M}(i)=\sum_{j=1}^{\infty} L(i, j) \operatorname{tr}\left(\rho_{i} M_{j}\right)
$$

The expectation of the risk function is called the Bayes risk, and denoted by $r(\mathbb{M}, \pi)$, i.e.

$$
r(\mathbb{M}, \pi)=\sum_{i=1}^{\infty} \sum_{j=1}^{\infty} \pi_{i} L(i, j) \operatorname{tr}\left(\rho_{i} M_{j}\right) .
$$

Consider the concrete loss function of the form

$$
L(i, j)=1-\delta_{i j} .
$$

Then we have

$$
r(\mathbb{M}, \pi)=\sum_{i=1}^{\infty} \sum_{j=1}^{\infty} \pi_{i}\left(1-\delta_{i j}\right) \operatorname{tr}\left(\rho_{i} M_{j}\right)=1-\sum_{i=1}^{\infty} \pi_{i} \operatorname{tr}\left(\rho_{i} M_{i}\right) .
$$

In this case, minimizing Bayes risk is equivalent to maximizing the expression

$$
\sum_{i=1}^{\infty} \pi_{i} \operatorname{tr}\left(\rho_{i} M_{i}\right)
$$

The above expression is the probability of correct guess while performing measurement $\mathbb{M}$, called the probability of detection. We shall denote this probability by $\mathbb{P}_{D}(\mathbb{M})$. We want to find a measurement which maximizes the probability of detection.

The existence of an optimal measurement is discussed in [7] in a general setup. In our case, the following result from [7] is sufficient.

Theorem 1 There exists a measurement maximizing the probability of detection.

For two states the solution can be achieved by taking the simple measurement made by the projections on the support of the positive and negative part of the Hermitian operator $\pi_{1} \rho_{1}-\pi_{2} \rho_{2}$. Kaniowski [4] did a deeper analysis for two finite dimensional projections with arbitrary a priori probability.

Each state $\rho_{i}$ has the spectral decomposition

$$
\rho_{i}=\sum_{j=1}^{m_{i}} \lambda_{i}^{j}\left|\varphi_{i}^{j}\right\rangle\left\langle\varphi_{i}^{j}\right|
$$

where $\lambda_{i}^{j}>0$ and $m_{i} \in\{1,2, \ldots, \infty\}$. In our further considerations we assume that the vectors $\left\{\varphi_{n}^{m}\right\}$ span the Hilbert space $\mathcal{H}$. For arbitrary states it is hard to say anything about the optimal measurement. In the case of finite-dimensional Hilbert space and finite number of a states it is natural to assume linear independence of vectors $\left\{\varphi_{n}^{m}\right\}$. Then we say that the states are linearly independent. For $\operatorname{dim} \mathcal{H}=\infty$ we have a stronger assumption. We say that states are strongly linearly independent if vectors $\left\{\varphi_{i}^{j}\right\}$ are strongly linearly independent, i.e. for each $i, j$ we have $\varphi_{i}^{j} \notin \overline{\operatorname{Lin}\left\{\varphi_{n}^{m}: n \neq i, m \neq j\right\}}$. 
A state $\rho$ is called pure if it has the form $|\varphi\rangle\langle\varphi|$ for some unit vector $\varphi \in \mathcal{H}$, otherwise a state is called mixed. For $\operatorname{dim} \mathcal{H}<\infty$ and pure states Kennedy [5, 6] obtained the following result.

Theorem (Kennedy [5, 6], 1973,74) Let pure states $\rho_{1}, \rho_{2}, \ldots, \rho_{n}$ be linearly independent. Then there exists a unique measurement maximizing the probability of detection and this measurement is simple.

It turns out that this result holds also for $\operatorname{dim} \mathcal{H}=\infty$.

Theorem (Luczak [7], 2009) Let pure states $\rho_{1}, \rho_{2}, \ldots$ be strongly linearly independent. Then there exists a unique measurement maximizing the probability of detection and this measurement is simple.

For $\operatorname{dim} \mathcal{H}<\infty$ and arbitrary states Eldar [2] obtained the following result.

Theorem (Eldar [2], 2003) Let the states $\rho_{1}, \rho_{2}, \ldots, \rho_{n}$ be linearly independent. Then there exists a unique measurement maximizing the probability of detection and this measurement is simple.

A natural question is whether the Eldar result can be generalized to infinite dimension. In this paper we show that the answer is positive.

\section{Optimal Measurement}

From Theorem 1 there exists a measurement which maximize the probability of detection. One of the most useful optimal measurement conditions was obtained in [3, Theorem II.2.2] and says that

Theorem (Holevo condition) Let $\mathbb{M}=\left(M_{1}, M_{2}, \ldots\right)$ be an optimal measurement for the probability of detection. Then the operator $\Lambda=\sum_{i} \pi_{i} \rho_{i} M_{i}$ is Hermitian and

$$
\left(\Lambda-\pi_{i} \rho_{i}\right) M_{i}=0 \quad \text { for all } i^{\prime} s
$$

Let $\rho_{1}, \rho_{2}, \ldots$ be states of the the form (1). Assume that for some $k$ we have

$$
\varphi_{k}^{j} \notin \overline{\operatorname{Lin}\left\{\varphi_{n}^{m}: n \neq k, m \neq j\right\}}, j=1,2, \ldots, m_{k} .
$$

Lemma 1 If $\mathbb{M}=\left(M_{1}, M_{2}, \ldots\right)$ is an optimal measurement, then for each $j^{\prime} s$

$$
M_{k} \varphi_{k}^{j} \neq 0
$$

Proof We use the method from the proof of Lemma 4 in [7]. Assume that we have e.g., $M_{1} \varphi_{1}^{1}=0$. Let $Q$ be a projection onto

$$
\overline{\operatorname{Lin}\left(\left\{\varphi_{i}^{j}: j=1,2, \ldots, m_{i}, i=1,2, \ldots\right\} \backslash\left\{\varphi_{1}^{1}\right\}\right)} .
$$


Define a new measurement $\hat{\mathbb{M}}=\left(\mathbb{1}-Q+Q M_{1} Q, Q M_{2} Q, Q M_{3} Q, \ldots\right)$. Since $Q \rho_{1} Q=$ $\sum_{k=2}^{m_{1}} \lambda_{1}^{k}\left|\varphi_{1}^{k}\right\rangle\left\langle\varphi_{1}^{k}\right|$ and $Q \rho_{i} Q=\rho_{i}, i=2,3, \ldots$, we have

$$
\begin{aligned}
\mathbb{P}_{D}(\hat{\mathbb{M}}) & =\pi_{1} \operatorname{tr}\left(\rho_{1}(\mathbb{1}-Q)\right)+\pi_{1} \operatorname{tr}\left(\rho_{1} Q M_{1} Q\right)+\sum_{i=2}^{\infty} \pi_{i} \operatorname{tr}\left(\rho_{i} Q M_{i} Q\right) \\
& =\pi_{1} \operatorname{tr}\left(\rho_{1}(\mathbb{1}-Q)\right)+\pi_{1} \operatorname{tr}\left(\sum_{k=2}^{m_{1}} \lambda_{1}^{k}\left|\varphi_{1}^{k}\right\rangle\left\langle\varphi_{1}^{k}\right| M_{1}\right)+\sum_{i=2}^{\infty} \pi_{i} \operatorname{tr}\left(\rho_{i} M_{i}\right) \\
& =\pi_{1} \operatorname{tr}\left(\rho_{1}(\mathbb{1}-Q)\right)+\mathbb{P}_{D}(\mathbb{M}) .
\end{aligned}
$$

From the above and the optimality of the measurement $\mathbb{M}$ we obtain that $\operatorname{tr}\left(\rho_{1}(\mathbb{1}-Q)\right)=0$. Therefore $\operatorname{tr}\left(\rho_{1} Q\right)=1$. This gives

$$
\sum_{j=1}^{m_{1}} \lambda_{1}^{j}\left\langle\varphi_{1}^{j} \mid Q \varphi_{1}^{j}\right\rangle=1 \Leftrightarrow \forall_{j=1,2, \ldots, m_{1}}\left\langle\varphi_{1}^{j} \mid Q \varphi_{1}^{j}\right\rangle=1 \Leftrightarrow \forall_{j=1,2, \ldots, m_{1}} Q \varphi_{1}^{j}=\varphi_{1}^{j} .
$$

This contradicts the relation

$$
\varphi_{1}^{1} \notin \overline{\operatorname{Lin}\left(\left\{\varphi_{i}^{j}: j=1,2, \ldots, m_{i}, i=1,2, \ldots\right\} \backslash\left\{\varphi_{1}^{1}\right\}\right)} .
$$

Before the main theorem we show an interesting result.

Theorem 2 If $\mathbb{M}=\left(M_{1}, M_{2}, \ldots\right)$ is an optimal measurement, then $M_{k}$ is a nonzero uniquely determined projection.

Proof From the Holevo condition we have

$$
\sum_{i \neq k} \pi_{i} \rho_{i} M_{i} M_{k}=\pi_{k} \rho_{k}\left(M_{k}-M_{k}^{2}\right) .
$$

Therefore for all $\xi \in \mathcal{H}$ we obtain

$$
\sum_{i \neq k} \sum_{j} \pi_{i} \lambda_{i}^{j}\left\langle\varphi_{i}^{j} \mid M_{i} M_{k} \xi\right\rangle \varphi_{i}^{j}=\sum_{j} \pi_{k} \lambda_{k}^{j}\left\langle\varphi_{k}^{j} \mid\left(M_{k}-M_{k}^{2}\right) \xi\right\rangle \varphi_{k}^{j} .
$$

From the above and assumption (2) on vectors $\left\{\varphi_{i}^{k}\right\}$ we have for all $\xi \in \mathcal{H}$ and $j^{\prime} s$

$$
\left\langle\varphi_{k}^{j} \mid\left(M_{k}-M_{k}^{2}\right) \xi\right\rangle=0
$$

so

$$
M_{k}^{2} \varphi_{k}^{j}=M_{k} \varphi_{k}^{j}
$$

Hence and from Lemma 1 there exists $j$ such that $M_{k} \varphi_{i}^{j}$ is an eigenvector of the operator $M_{i}$ with eigenvalue equal to 1 . Let $J_{k}$ be the set of all such $j^{\prime} s$.

Define a sequence $\hat{\mathbb{M}}=\left(\hat{M}_{1}, \hat{M}_{2}, \ldots\right)$ where $\hat{M}_{i}=M_{i}, i \neq k$ and $\hat{M}_{k}$ is a nonzero projection onto

$$
\overline{\operatorname{Lin}\left\{M_{k} \varphi_{k}^{j}: j \in J_{k}\right\}} \text {. }
$$

Because $\hat{M}_{k} \leq M_{k}$ we have $\operatorname{tr}\left(\rho_{k} \hat{M}_{k}\right) \leq \operatorname{tr}\left(\rho_{k} M_{k}\right)$. On the other hand, since $\hat{M}_{k}$ commutes with $M_{k}$

$$
\operatorname{tr}\left(\rho_{k} M_{k}\right)=\operatorname{tr}\left(\hat{M}_{k} M_{k} \rho_{k}\right)=\operatorname{tr}\left(\rho^{\frac{1}{2}} \hat{M}_{k} M_{k} \hat{M}_{k} \rho_{k}^{\frac{1}{2}}\right) \leq \operatorname{tr}\left(\rho^{\frac{1}{2}} \hat{M}_{k} \rho_{k}^{\frac{1}{2}}\right)=\operatorname{tr}\left(\rho_{k} \hat{M}_{k}\right) .
$$


These inequalities give $\operatorname{tr}\left(\rho_{k} M_{k}\right)=\operatorname{tr}\left(\rho_{k} \hat{M}_{k}\right)$, hence

$$
\mathbb{P}_{D}(\hat{\mathbb{M}})=\mathbb{P}_{D}(\mathbb{M}) \text {. }
$$

Assume that $T=\mathbb{1}-\sum_{i=1}^{\infty} \hat{M}_{i}$ is a nonzero operator. Then there exist numbers $i, j$ such that $\left\langle\varphi_{i}^{j} \mid T \varphi_{i}^{j}\right\rangle>0$ because vectors $\left\{\varphi_{n}^{m}\right\}$ span the space $\mathcal{H}$. Define a new measurement $\hat{\mathbb{N}}=\left(\hat{M}_{1}, \hat{M}_{2}, \ldots, \hat{M}_{i}+T, \ldots\right)$. From (3) we have

$$
\mathbb{P}_{D}(\hat{\mathbb{N}})=\sum_{n=1}^{\infty} \pi_{n} \operatorname{tr}\left(\rho_{n} \hat{M}_{n}\right)+\pi_{i} \operatorname{tr}\left(\rho_{i} T\right)=\mathbb{P}_{D}(\hat{\mathbb{M}})+\pi_{i} \operatorname{tr}\left(\rho_{i} T\right)>\mathbb{P}_{D}(\mathbb{M}),
$$

which is impossible. Therefore $T=0$ and $\sum_{i=1}^{\infty} \hat{\mathbb{M}}_{i}=\mathbb{1}$. Hence $M_{k}=\hat{M}_{k}$.

Assume that $\mathbb{M}=\left(M_{1}, M_{2}, \ldots\right)$ and $\mathbb{N}=\left(N_{1}, N_{2}, \ldots\right)$ are two distinct optimal measurements such that $M_{k} \neq N_{k}$. From the above $M_{k}$ and $N_{k}$ are projections. Of course $\frac{1}{2} \mathbb{M}+\frac{1}{2} \mathbb{N}$ is also an optimal measurement and $\frac{1}{2} M_{k}+\frac{1}{2} N_{k}$ is a projection. Then

$$
\begin{array}{r}
\frac{1}{2} M_{k}+\frac{1}{2} N_{k}=\left(\frac{1}{2} M_{k}+\frac{1}{2} N_{k}\right)^{2} \Leftrightarrow \\
2 M_{k}+2 N_{k}=M_{k}+M_{k} N_{k}+N_{k} M_{k}+N_{k} \Leftrightarrow \\
\left(M_{k}-N_{k}\right)^{2}=0 \Leftrightarrow M_{k}=N_{k},
\end{array}
$$

a contradiction. Consequently, $M_{k}$ is uniquely determined.

Let the states $\rho_{1}, \rho_{2}, \ldots$ of the form (1) be strongly linearly independent. Our main theorem is

Theorem 3 There exists a unique measurement maximizing the probability of detection and this measurement is simple with the nonzero outcomes.

Proof Let $\mathbb{M}=\left(M_{1}, M_{2}, \ldots\right)$ be a measurement maximizing the probability of detection. From Theorem 2 each $M_{i}$ is a nonzero uniquely determined projection. Of course all $M_{i}$ are mutually orthogonal because

$$
M_{1}+M_{2}+\ldots=\mathbb{1} .
$$

Therefore $\mathbb{M}$ is the unique simple measurement with the nonzero outcomes.

Suppose now that $\rho_{1}, \rho_{2}, \ldots$ are arbitrary states linearly independent or not. The next theorem shows a relation between the ranges of elements of an optimal measurement and the ranges of the states in question.

Theorem 4 Let $\mathbb{M}=\left(M_{1}, M_{2}, \ldots\right)$ be a measurement maximizing the probabilty of detection. Then

$$
\text { dimRange } M_{i} \leq{\text { dimRange } \rho_{i}}
$$

for all $i^{\prime} s$.

Proof Let $\Lambda$ be the operator in the Holevo condition. From this condition we have

$$
\left(\Lambda-\pi_{i} \rho_{i}\right) M_{i}=0 \quad \text { for all } i^{\prime} s .
$$


Let $\xi$ be an arbitrary nonzero vector in $\mathcal{H}$. Then exists $k, j$ such that

$$
\left\langle\xi \mid \varphi_{k}^{j}\right\rangle \neq 0
$$

because vectors $\left\{\varphi_{n}^{m}\right\}$ span the space $\mathcal{H}$. From [3, Theorem II.2.2] we obtain

$$
\Lambda \geq \pi_{i} \rho_{i} \quad \text { for all } i^{\prime} s \text {. }
$$

Therefore

$$
\langle\xi \mid \Lambda \xi\rangle \geq_{(6)}\left\langle\xi \mid \pi_{k} \rho_{k} \xi\right\rangle \geq \pi_{k}\left\langle\xi \mid \varphi_{k}^{j}\right\rangle\left\langle\varphi_{k}^{j} \mid \xi\right\rangle>_{(5)} 0,
$$

so the operator $\Lambda$ is invertible.

Consequently, condition (4) is of the form

$$
M_{i}=\pi_{i} \Lambda^{-1} \rho_{i} M_{i} .
$$

This implies

$$
\text { dimRange } M_{i} \leq \operatorname{dimRange} \rho_{i} .
$$

As a corollary we obtain the main result of [7].

Corollary 1 Let pure states $\rho_{1}, \rho_{2}, \ldots$ be strongly linearly independent. Then there exists a unique measurement maximizing the probability of detection and this measurement is simple. The outcomes of this measurement are rank one operators.

Proof The first part of the corollary is a consequence of Theorem 3. From Theorem 4 the outcomes of the optimal measurement are zero or rank one operators but Lemma 1 implies that the outcomes can't be zero operators.

Acknowledgments The author would like to thank A. Łuczak for valuable comments which helped to improve the final version of the paper.

Open Access This article is distributed under the terms of the Creative Commons Attribution License which permits any use, distribution, and reproduction in any medium, provided the original author(s) and the source are credited.

\section{References}

1. Busch, P., Grabowski, M., Lahti, P.J.: Operational Quantum Physics. Springer-Verlag, Berlin (1995)

2. Eldar, Y.C.: Von Neumann measurement is optimal for detecting linearly independent mixed quantum state. Phys. Rev. A 68, 052303:1-052303:4 (2003)

3. Holevo, A.S.: Investigations in the general theory of statistical decisions. In: Proceedings of the Steklov Institute of Mathematics, vol. 124, issue 3 (1978)

4. Kaniowski, K.: Optimal ambiguous discrimination between the states given by two projections. Math. Slovaca 64, 247-258 (2014)

5. Kennedy, R.S.: On the optimum receiver for the M-ary linearly independent pure state problem . M.I.T Res. Lab. Electron. Quart. Prog. Rep 110, 142 (1973)

6. Kennedy, R.S.: Uniqueness of the optimum receiver for the M-ary pure state problem. M.I.T. Res. Lab. Electron. Quart. Prog. Rep 113, 129 (1974)

7. Łuczak, A.: Maximizing the probability of detection for pure states. J. Math. Phys. 50, 053502:1053502:14 (2009) 